\title{
Ceramic Nanocrystalline Superconductor Gadolinium Barium Copper Oxide (GdBaCu0) at Different Treating Temperatures
}

\author{
V. S. Vinila ${ }^{1}$, Reenu Jacob ${ }^{2}$, Anusha Mony ${ }^{1}$, Harikrishnan G. Nair ${ }^{1}$, Sheelakumari Issac ${ }^{3}$, \\ Sam Rajan², Anitha S. Nair ${ }^{4}$, D. J. Satheesh'1, Jayakumari Isac ${ }^{*}$ \\ ${ }^{1}$ Centre for Condensed Matter, Department of Physics, CMS College, Kottayam, India \\ ${ }^{2}$ Department of Physics, CMS College, Kottayam, India \\ ${ }^{3}$ Department of Chemistry, UC College, Aluva, India \\ ${ }^{4}$ Department of Physics, DB College, Parumala, India \\ Email: ${ }^{*}$ drjayacmscollege@gmail.com, ${ }^{*}$ jayacmscollege@gmail.com
}

Received 24 May 2014; revised 24 June 2014; accepted 24 July 2014

Copyright @ 2014 by authors and Scientific Research Publishing Inc.

This work is licensed under the Creative Commons Attribution International License (CC BY).

http://creativecommons.org/licenses/by/4.0/

(c) (i) Open Access

\section{Abstract}

With the discovery of high-TC superconducting materials like Yttrium Barium Cupric Oxide, Bismuth Strontium Calcium Copper Oxide and Thallium Calcium Barium Copper Oxide, tremendous interest has developed over the past two years in understanding these materials as well as utilizing them in a variety of applications. The thin films of these materials are expected to play an important role in the area of microelectronics, especially for interconnects in integrated circuits, Josephson junctions, magnetic field sensors and optical detectors. Here, the authors designed a new nanocrystalline ceramic type II high-TC superconductor, Gadolinium Barium Copper Oxide (GdBaCu0/GBCO). The GBCO perovskite phase structure was prepared by the conventional solid state thermochemical reaction technique involving mixing, milling, calcination and sintering. In GBCO system, the method for controlling microstructure and superconducting state is related to oxygen content consideration because small changes in oxygen concentration can often lead to huge change in Tc. In order to show the viability of the proposed method, super-conducting powder was prepared in special furnace. The sample was analyzed by X-Ray Diffraction (XRD), an indispensible non-destructive tool for structural materials characterization and quality control which makes use of the Debye-Scherrer method. The comparison of XRD results with JCPDS files confirmed the orthorhombic structure of the sample. Micro-structural features are studied using Scanning Electron Microscopy (SEM) which revealed that its particle size is in the nanometer range. It also confirmed the calculated value of particle size from Debye Scherrer's formula. EDX plot shows the presence of all the constituents. X-ray instrumental peak broadening analysis was used to evaluate the size and lattice strain by the Williamson-Hall Plot method.

\footnotetext{
*Corresponding author.
}

How to cite this paper: Vinila, V.S., Jacob, R., Mony, A., Nair, H.G., Issac, S., Rajan, S., Nair, A.S., Satheesh, D.J. and Isac, J. (2014) Ceramic Nanocrystalline Superconductor Gadolinium Barium Copper Oxide (GdBaCuO) at Different Treating Temperatures. Journal of Crystallization Process and Technology, 4, 168-178. http://dx.doi.org/10.4236/jcpt.2014.43021 


\section{Keywords}

\section{Gadolinium Barium Copper Oxide (GBC0), Nanocrystalline, XRD, SEM, EDX, Debye Scherrer's Formula, Instrumental Broadening, Williamson-Hall Plot Method}

\section{Introduction}

Ceramic materials are inorganic, non-metallic materials and things made from them. They may be crystalline or partly crystalline. They are formed by the action of heat and subsequent cooling. They are brittle, hard and strong in compression, and weak in shearing and tension. They withstand chemical erosion that occurs in an acidic or caustic environment. Ceramics generally can withstand very high temperatures such as temperatures that range from $1000^{\circ} \mathrm{C}$ to $1600^{\circ} \mathrm{C}\left(1800^{\circ} \mathrm{F}\right.$ to $\left.3000^{\circ} \mathrm{F}\right)$. The Gadolinium Barium Copper Oxide (GBCO) is a type of cuprate ceramic superconductor. All superconducting cuprates are layered materials having a complex structure described as a superlattice of superconducting $\mathrm{CuO}_{2}$ layers separated by spacer layers where the misfit strain between different layers and dopants in the spacers induces a complex heterogeneity which is intrinsic for high-temperature superconductivity in the superstripes scenario. A new era in the study of superconductivity began in 1986 with the discovery of high critical temperature superconductor and introduced the outstanding challenges of theoretical condensed matter physics [1]. Since there is a complete expulsion of magnetic flux, superconductors are perfectly diamagnetic [2] [3]. High-voltage generator can be developed by using HTS inductor and electronic RCL series resonant circuit [4] [5]. Before final heating at $900^{\circ} \mathrm{C}$, the material $\mathrm{GBCO}$ is treated at different temperatures, $30^{\circ} \mathrm{C}, 500^{\circ} \mathrm{C}, 850^{\circ} \mathrm{C}$ and $900^{\circ} \mathrm{C}$.

In this work the authors describe the preparation of GBCO ceramic material and it is characterized to show good quality, homogeneity and the desired stoichiometry of the sample prepared. The results were analyzed by X-Ray Diffraction (XRD), SEM, and EDX. The particle size was determined from XRD details by Debye Scherrer formula. The SEM studies revealed that its particle size is in hundred-nanometer range. The EDX spectrum of GBCO gave the information on the elemental composition of the material. The particle size and strain of the material were found by Instrumental Broadening and Williamson-Hall Plot method.

\section{Materials and Methods}

GBCO has perovskite structure. The perovskite structure is adopted by many oxides that have the chemical formula $\mathrm{ABO}_{3}$ [6]. The representative structure of perovskite compounds is cubic, the compounds in this family may possess some distortion. The orthorhombic and tetragonal phases are most common variants. The sample with the chemical formula $\mathrm{GdBa}_{2} \mathrm{Cu}_{3} \mathrm{O}_{7-\mathrm{x}}$ (Figure 1) was prepared by the conventional solid state reaction tech-

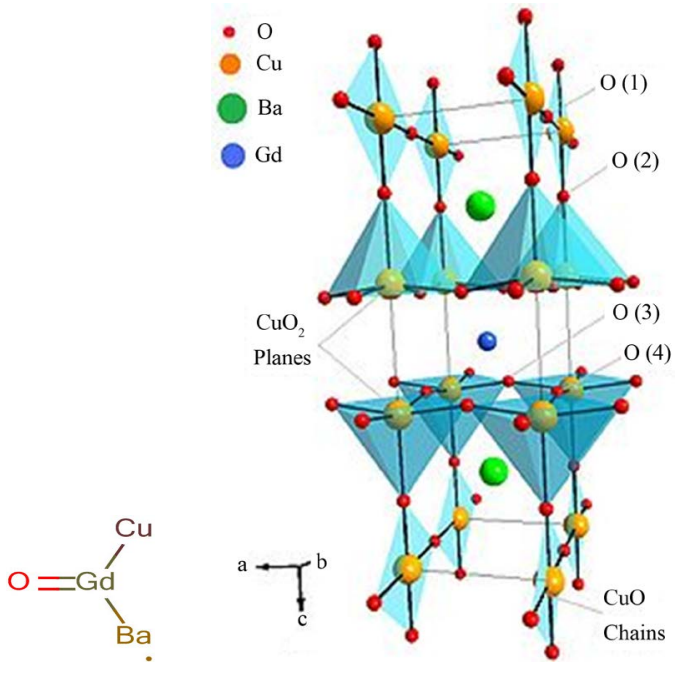

Figure 1. GdBCO unit cell (CAS No. 110687-67-7). 
nique according to the molecular formula. For the prepared sample, the reagent grade chemicals of high purity (99.99\%) $\mathrm{Gd}_{2} \mathrm{O}_{3}, \mathrm{BaCO}_{3}$ and $\mathrm{CuO}$ powders were used as the raw materials and weighed according to their molecular formula.

The powders of the required ceramics were mixed mechanically. Mechanical mixing is usually done by hand mixing in agate mortar for very long time. Then ball milled with suitable balls to insure homogeneity and milling. Then the material calcined at different temperatures, $30^{\circ} \mathrm{C}, 500^{\circ} \mathrm{C}, 850^{\circ} \mathrm{C}$ and $900^{\circ} \mathrm{C}$. After the furnace is off, on cooling the oxygen is allowed to flow into the furnace at intervals (oxygen annealing). A final furnace temperature of $900^{\circ} \mathrm{C}$ is maintained for the intermediate firings. A temperature much higher than this will result in a material that is much harder to regrind. Temperatures above $1030^{\circ} \mathrm{C}$ may destroy the crystal structure. Then $\mathrm{X}$-ray diffraction spectrum of these materials was taken. For experimental conformation of the calculated particle size value, Scanning Electron Microscopy (SEM) photograph was taken. From EDX, the composition details of the prepared ceramics were determined.

\section{Results and Discussion}

\subsection{XRD Analysis}

X-ray diffraction pattern for the four different temperatures in steps for the sample was taken using Bruker AXS D8 advance diffractometer. The diffractometer with radiations of wavelength $1.54184 \AA$ having Nickel filter, equipped with X-ray generator 1140/90/96 having X-ray source KRISTALLOFLXE 780 KF, 4 KE with wide angle goniometer PW1710/70 with single pen recorder pm 8203 and channel control PW1390 at 35 kV, $10 \mathrm{~mA}$ is used for the purpose. The scanning speed of the specimen is 2 degree/minute. From the XRD results, the obtained d values matching with the JCPDS (Joint Committee on Powder Diffraction Standards) file values. So that it can be concluded that this crystal is found to be orthorhombic system:

$$
A=3.837, b=3.677, c=11.786, \alpha=\beta=\gamma=90^{\circ}
$$

From the XRD data, we can calculate the particle size of GBCO by using Debye Scherrer equation. Here the authors studied the change of XRD spectrum of GBCO at different treating temperatures.

The XRD patterns of GBCO powder obtained for various annealing temperatures are shown in Figure 2. XRD spectrum for the different temperatures gave a clear idea about the maximum intensity peak shifting corresponds to the different treating temperatures. And also get the maximum intensity peak difference. The temperature increases, the highest peaks in the XRD spectrums shifts from left to right through the $2 \theta$ axis and the highest intensity peak decreases.

The atoms undergo thermal vibration about their mean positions even at the absolute zero of temperature, and the amplitude of this vibration increases as the temperature increases. Increased thermal vibration of the atoms, as the result of an increase in temperature, the unit cell expands, causing changes in plane spacing $d$ and therefore in the $2 \theta$ positions of the diffraction lines. And also the intensities of the diffraction lines decrease.

When a material heated from room temperature to high temperature, the amplitude of the thermal vibrations increased. The amplitude is very higher than that at room temperature. After heating, the material cooled to room temperature. The amplitude of the atomic vibrations of the material was decreased. But it couldn't arrive at the initial amplitude. The atomic vibration amplitude of the heated material was higher than initial amplitude. That means, the atomic vibration amplitude increases, the intensity of the diffracted beam also decreases because it has the effect of smearing out lattice planes. Thus the reinforcement of waves scattered at the Bragg angle by various parallel planes is not as perfect as it is for a crystal with fixed atoms. This reinforcement requires that the path difference, which is a function of the plane spacing $d$, between waves scattered by adjacent planes be an integral number of wavelengths. The thickness of the planes is $2 u$, where $u$ is the average displacement of an atom from its mean position. Under these conditions reinforcement is no longer perfect, and it becomes more imperfect as the ratio $u / d$ increases, i.e., as the temperature increases since that increases $u$, or as $\theta$ increases, since high- $\theta$ reflections involve planes of low $d$ value. Thus the intensity of a diffracted beam decreases as the temperature is raised. In intensity calculations, the temperature factor $\mathrm{e}^{-2 \mathrm{M}}$ decreases as $2 \theta$ increases [7].

\subsection{Particle Size Measurements}

Crystal structures are solved by analyzing the intensities of diffracted X-ray beams. X-ray diffraction profile may be used to measure the average crystal size in the sample provided the average diameter is less than $200 \AA$. 
V. S. Vinila et al.

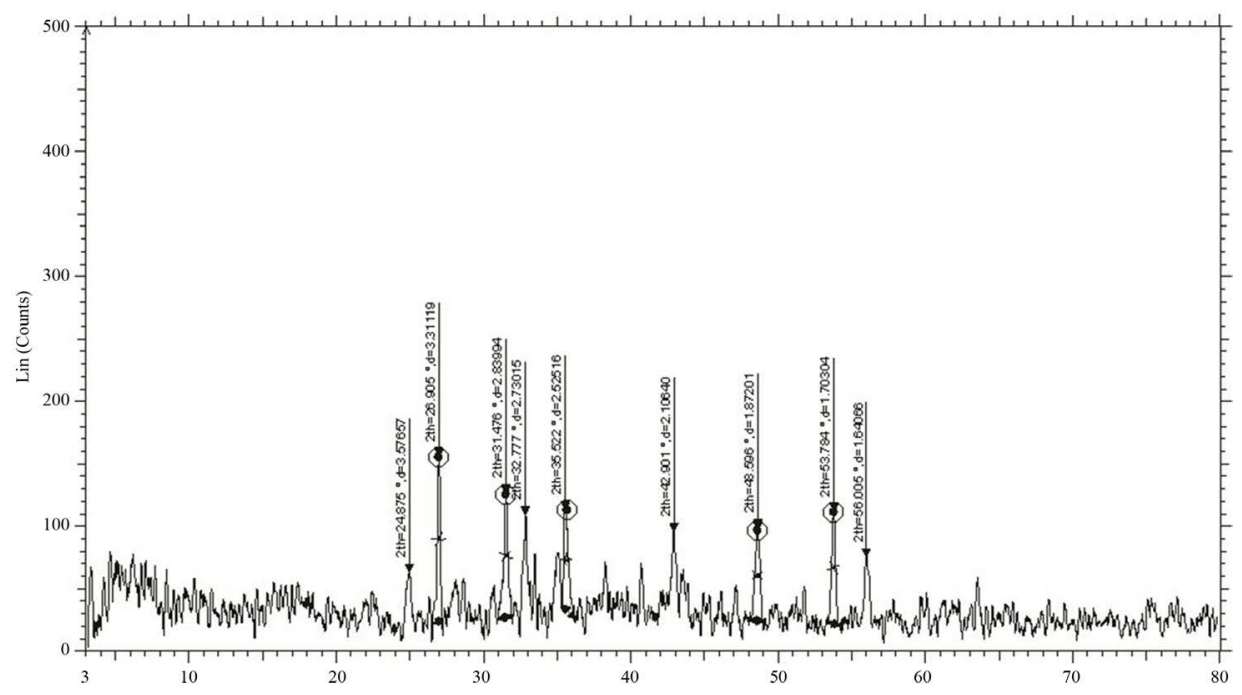

(a)

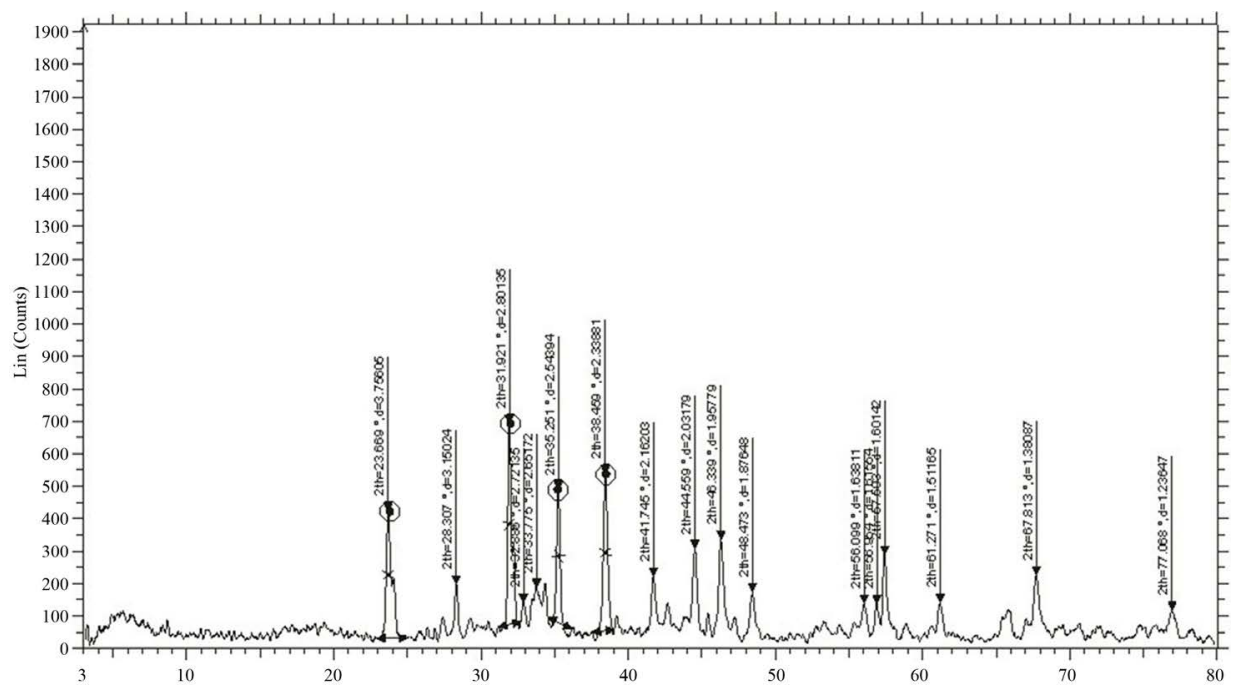

(b)

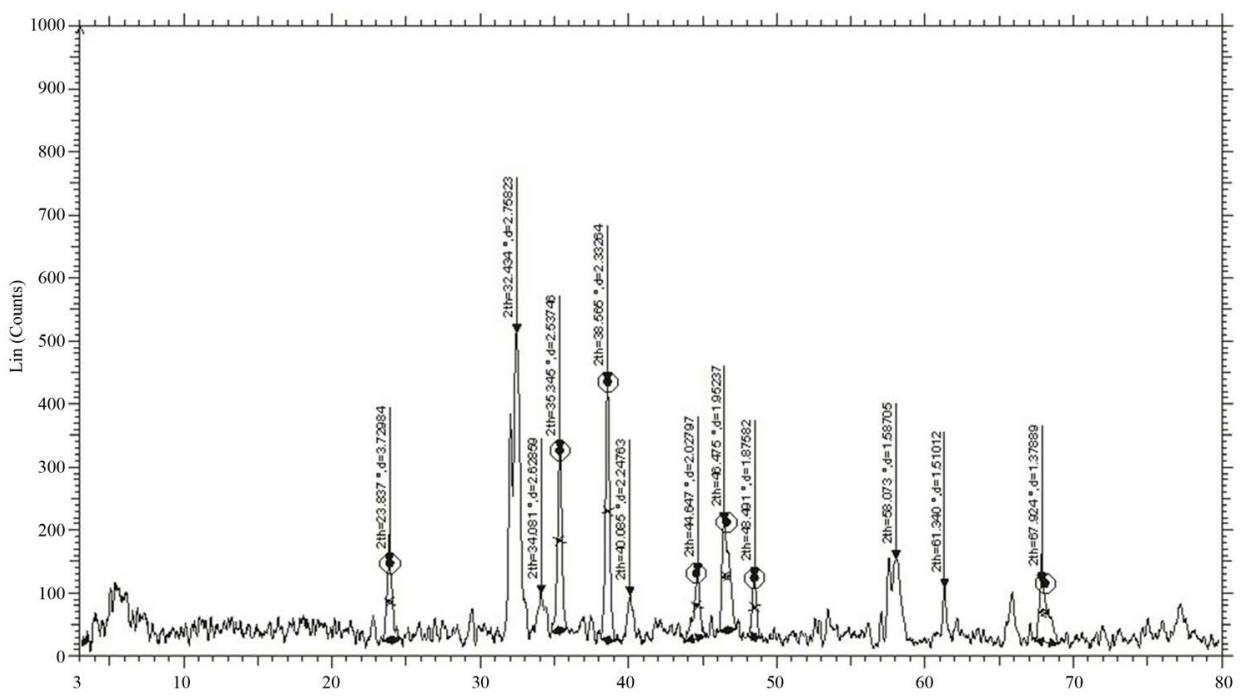

(c)

(171) 


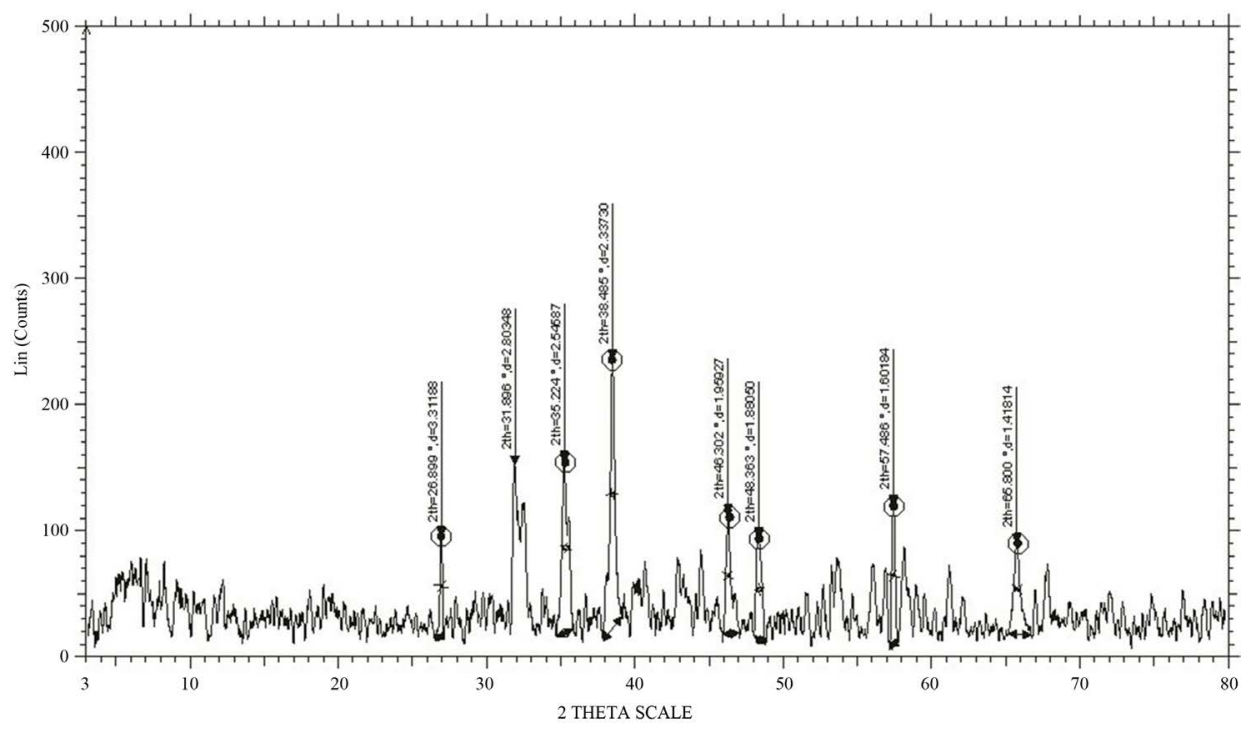

(d)

Figure 2. XRD of GBCO at temperatures (a) $30^{\circ} \mathrm{C}$, (b) $500^{\circ} \mathrm{C}$, (c) $850^{\circ} \mathrm{C}$ and (d) $900^{\circ} \mathrm{C}$.

The lines in a powder diffraction pattern are of finite breadth but if the particles are very small, the lines are broaden than usual. The broadening decreases with the increase in particle size. The particle size for GBCO was calculated from X-ray diffraction profiles of strong reflections with intensity\% by measuring the Full Width at Half Maximum (FWHM). The Debye Scherrer equation for calculating the particle size is given by [8]

$$
D=\frac{K \lambda}{\beta \cos \theta}
$$

where $K$ is the Scherrer constant, $\lambda$ is the wavelength of light used for the diffraction, $\beta$ is the "full width at half maximum" of the sharp peaks, and $\theta$ is the angle measured. The Scherrer constant $(K)$ in the above formula accounts for the shape of the particle and is generally taken to have the value 0.9. The results reveal that the particle size is less than $100 \mathrm{~nm}$. The particle size of GBCO at different temperatures is shown in the Table 1.

From Figure 2, the peak broadening in the XRD patterns clearly indicated the nature of the very small nanocrystals. From the width of the XRD peak, the mean crystalline size can be calculated using Debye Scherrer's equation. From Table 1, particle size of the material GBCO increases with respect to the treating temperature increasing. And also it is realized that, $\theta$ increases with temperature. The results revealed that the particle size is less than $100 \mathrm{~nm}$. The diffraction data revealed that the material belongs to orthorhombic symmetry. The results were compared with JCPDS data.

Heat treatment causes the particles to anneal and form larger grains, which of course indicates that the particles become larger. Hence, the large particle size of sample at $900^{\circ} \mathrm{C}$ is expected. This also agrees with the higher crystallinity, as having larger grains means more long-range order, and hence more crystallinity [9] [10].

\subsection{XRD-Instrumental Broadening}

When particle size is less than $100 \mathrm{~nm}$, appreciable broadening in X-ray diffraction lines will occur. Diffraction pattern will show broadening because of particle size and strain. The observed line broadening will be used to estimate the average size of the particles. The total broadening of the diffraction peak is due to sample and the instrument. The sample broadening is described by

$$
F W(s) \times \cos \theta=\frac{K \times \lambda}{\text { size }}+4 \times \text { strain } \times \sin \theta
$$

The total broadening $\beta_{t}$ equation is described by

$$
\beta_{t}^{2} \approx\left\{\frac{0.9 \lambda}{D \cos \theta}\right\}^{2}+\{4 \varepsilon \tan \theta\}^{2}+\beta_{0}^{2}
$$


Table 1. The particle size of GBCO at different temperatures, calculated by Debye Scherrer formula.

\begin{tabular}{cccc}
\hline Data of high intensity peak of XRD at different temperatures & $\theta$ (degree) & $\beta$ (radian) $\left(\times 10^{-3}\right)$ & Particle size $(\mathrm{nm})$ \\
\hline GBCO-30 $0^{\circ} \mathrm{C}$ & 13.451 & 5.46 & 25.42 \\
GBCO-500 ${ }^{\circ} \mathrm{C}$ & 15.963 & 5.22 & 27.64 \\
GBCO-850 ${ }^{\circ} \mathrm{C}$ & 16.217 & 4.13 & 33.60 \\
GBCO-900 ${ }^{\circ} \mathrm{C}$ & 19.245 & 3.80 & 36.52 \\
\hline
\end{tabular}

where $D$ is average particle size, $\varepsilon$ is strain and $\beta_{0}$ is instrumental broadening. Instrumental broadening is presented in Figure 3.

The size and strain of the experimentally observed broadening of several peaks are computed simultaneously using least squares method. When, particle size becomes smaller, due to size effect, the peaks become broad and widths larger. The broadening of peak may also occur due to micro strains of the crystal structure arising from defects like dislocation and twinning [11].

\subsection{Williamson-Hall Plot}

This method is attributed to G. K. Williamson and his student, W. H. Hall. It relies on the principle that the approximate formulae for size broadening, $\beta_{L}$, and strain broadening, $\beta_{e}$, vary quite differently with respect to Bragg angle, $\theta$. Williamson and Hall proposed a method for deconvoluting size and strain broadening by looking at the peak width as a function of $2 \theta[12]$.

$$
\begin{aligned}
& \beta_{L}=\frac{K \lambda}{L \cos \theta} \\
& \beta_{e}=C \varepsilon \tan \theta
\end{aligned}
$$

One contribution varies as $1 / \cos \theta$ and the other as $\tan \theta$. If both contributions are present then their combined effect should be determined by convolution. The simplification of Williamson and Hall is to assume the convolution is either a simple sum or sum of squares. Using the former equations, we get:

$$
\beta_{\text {tot }}=\beta_{e}+\beta_{L}=C \varepsilon \tan \theta+\frac{K \lambda}{L \cos \theta}
$$

If we multiply this equation by $\cos \theta$ we get:

$$
\beta_{\text {tot }} \cos \theta=C \varepsilon \sin \theta+\frac{K \lambda}{L}
$$

and comparing this to the standard equation for a straight line ( $m=$ slope; $c=$ intercept) $y=m x+c$.

We see that by plotting $\beta_{\text {tot }} \cos \theta$ versus $\sin \theta$ we obtain the strain component from the slope $(C \varepsilon)$ and the size component from the intercept $(K \lambda / L)$. Such a plot is known as a Williamson-Hall Plot. However the Williamson-Hall method has many assumptions: its absolute values should not be taken too seriously but it can be a useful method if used in the relative sense; for example a study of many powder patterns of the same chemical compound, but synthesized under different conditions, might reveal trends in the crystallite size/strain which in turn can be related to the properties of the product. Figure 4 shows Williamson Hall Plot.

Here, Williamson-Hall Plot is plotted with $\sin \theta$ on the $\mathrm{x}$-axis and $\beta \cos \theta$ on the $\mathrm{y}$-axis (in radians). A linear fit is got for the data. From this fit, particle size and strain are extracted from y-intercept and slope respectively. The extracted particle size is $35.01 \mathrm{~nm}$ and strain is 0.00488 from Williamson Hall Plot (Figure 4).

\subsection{SEM Analysis}

Morphology has been analyzed from JEOL Model JSM-6390LV Scanning Electron Microscope (SEM). The SEM uses an electron beam for surface imaging. The advantages of SEM over light microscopy are greater magnification and much larger depth of field. Different elements and surface topographies emit different quan- 


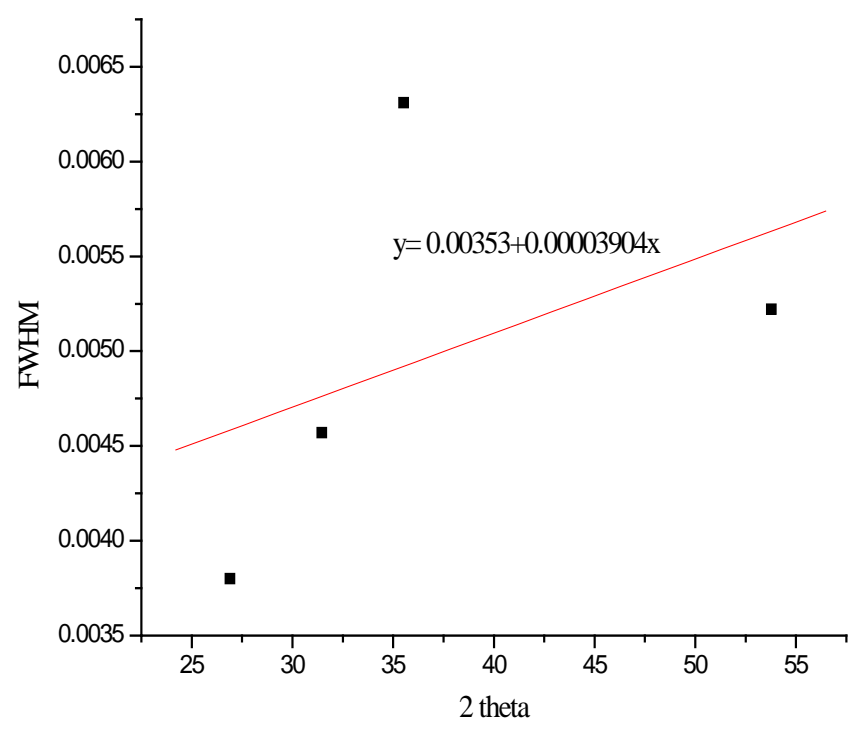

Figure 3. Typical instrumental broadening.

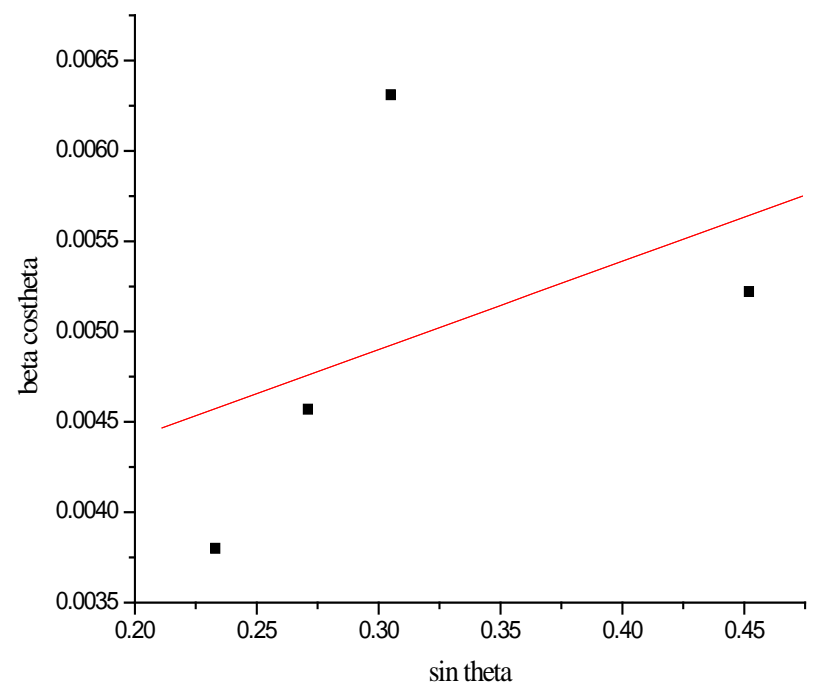

Figure 4. Williamson-Hall Plot. Slope $=0.00488$; Y intercept $=0.00442$; Correlation coefficient $=0.44011$; Standard deviation of the fit $=0.00117$.

tity of electrons, due to which the contrast in a SEM micrograph (picture) is representative of the surface topography and distribution of elemental composition on the surface. The SEM analyses the surface of solid objects, producing images of higher resolution than optical microscopy. It produces representations of three dimensional samples from a diverse range of materials. Figure 5 is the surface morphology of GBCO. The particle size measurement through SEM reveals its maximum dimensions always less than $100 \mathrm{~nm}$.

Figure 5 shows SEM image of GBCO. The SEM photograph revealed maximum dimensions of the particles to be always less than $100 \mathrm{~nm}$. This is an experimental proof of the theoretical calculation of particle size by Debye Scherrer equation from XRD data.

\subsection{Energy Dispersed X-Ray Spectrograph (EDX)}

EDX (Figure 6) shows the composition details of the prepared ceramic powders. The instrument used for this measurement is ISIS Link Oxford Instrument UK. This technique generally associated with Scanning Electron Microscope (SEM). In this technique an electron beam of $10-20 \mathrm{KeV}$ strikes the surface of a sample which 


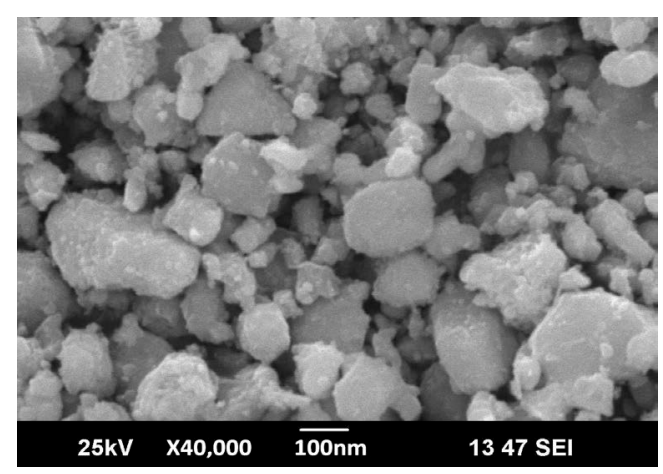

Figure 5. SEM photograph of GBCO.

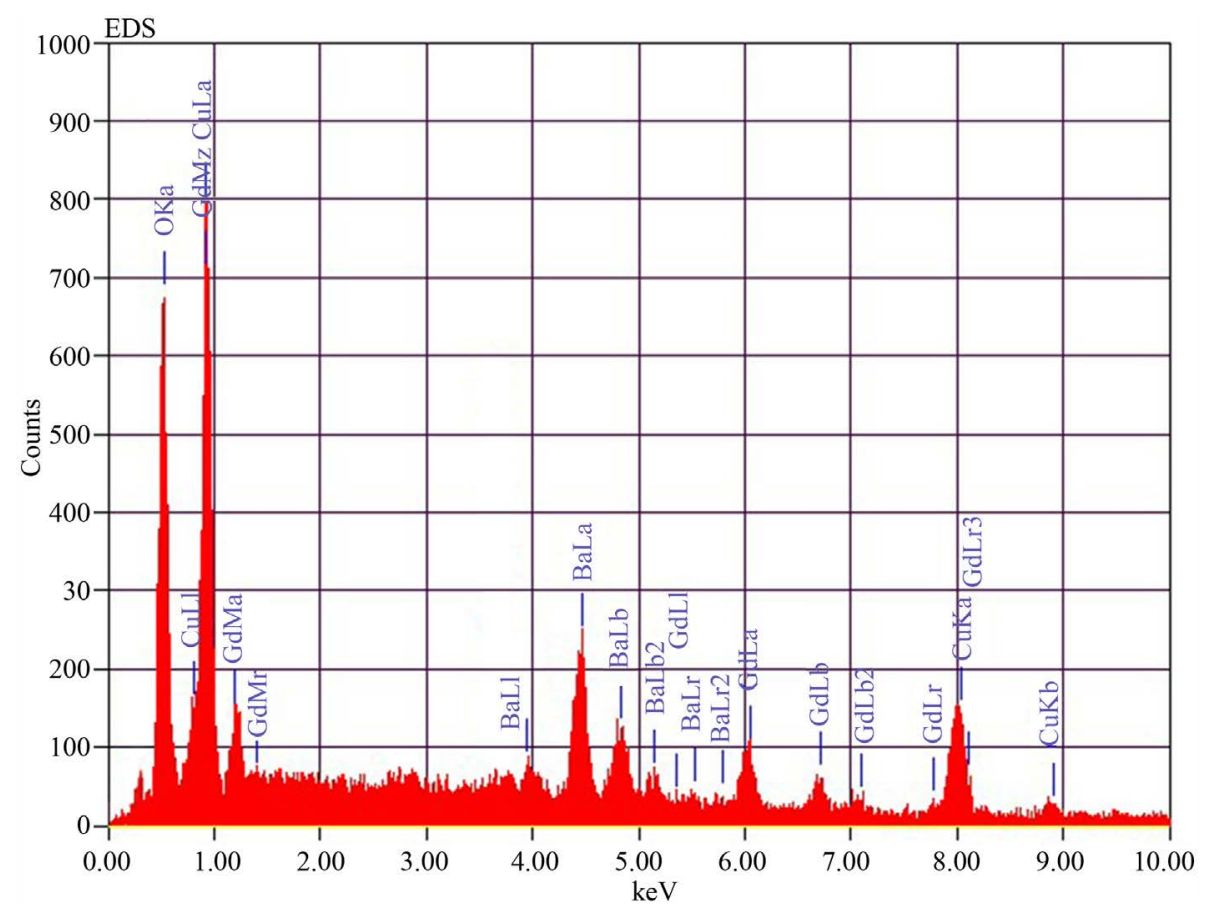

Figure 6. EDX of GBCO.

causes X-ray to be emitted from point of incidence. The energy of the X-ray emitted depends on material under examination. The energy of the characteristic X-ray emitted from the different elements is different and thus it gives the unavoidable signature of the particular element. When an X-ray strikes the detector, it will generate a photoelectron which in turn generates electron-hole pairs. A strong electric field attracts the electrons and holes towards the opposite ends of the detector. The size of the pulse thus generated depends on the number electron-hole pairs created, which in turn depends on the energy of the incoming X-ray. In this method however elements with low atomic number are difficult to be detected. The detector which is lithium doped silicon (SiLi) is protected by a beryllium window and operated at liquid nitrogen temperatures. The absorption of the soft $\mathrm{X}$-rays by the beryllium decreases the sensitivity below an atomic number of 11 .

EDX spectrum (Figure 6) of GBCO gave the information on the elemental composition of the material. The elemental compositions agree with the stoichiometric relations of the prepared compound. The EDX spectrums obtained give the material under investigation.

From the EDX spectrum, the four dominant peak positions at $6.056 \mathrm{keV}, 4.465 \mathrm{keV}, 8.04 \mathrm{keV}, 0.525 \mathrm{keV}$ correspond quite well to the energy pattern of the corresponding materials ( $\mathrm{Gd}, \mathrm{Ba}, \mathrm{Cu}$ and $\mathrm{O}$ ) reported in the EDAX international chart, giving the evidence that $\mathrm{Gd}, \mathrm{Cu}, \mathrm{Ba}$ and $\mathrm{O}$ are dominant in GBCO samples. Table 2 shows the percentage of the elements in the prepared GBCO sample. 
Table 2. Material content (EDX).

\begin{tabular}{cc}
\hline Material & Content (\%) \\
\hline Gd & 18.66 \\
Ba & 34.41 \\
$\mathrm{Cu}$ & 28.05 \\
$\mathrm{O}$ & 18.88 \\
\hline
\end{tabular}

\section{Conclusion}

In this work, GBCO ceramics were prepared successfully by the conventional solid state thermochemical reaction technique and characterized by XRD, SEM, EDX, and particle size measurement. XRD data confirmed the formation of the perovskite phase structure and the average particle size. The XRD spectrums of the GBCO at different temperatures indicate that, according to increasing treating temperature, the $\theta$ value and particle size also increase. From SEM analysis, the morphology images revealed the approximate size of the nanoparticles. The value of particle size calculated from the Williamson-Hall Plot method is in agreement with that of the particle size measured from Debye Scherrer formula. The EDX analysis indicates the presence of the elements existing in the sample and it agrees with the stoichiometric relations of the prepared compound.

\section{Acknowledgements}

The authors are thankful to Kerala State Council for Science, Technology and Environment (KSCSTE), Thiruvanathapuram for granting the financial assistance, SAIF, Cochin for providing instrumental help for the data analysis and to the Principal, CMS College, Kottayam, Kerala for providing the facilities.

\section{References}

[1] Definition of Superconductivity. http://www.wordiq.com/definition/Superconductivity

[2] Kittel, C. (2004) Introduction to Solid State Physics. 7th Edition, Wiley, India.

[3] Puri, R.K. and Babbar, V.K. (2009) Solid State Physics. S Chand \& Company Ltd., New Delhi.

[4] Narlikar, A.V. (2004) High Temperature Superconductivity. Springer, Berlin, 35.

[5] Jin, J.X. and Dou, S.X. (1999) Development of High TC Superconductors for Engineering Applications. Science \& Technology Advancing into New Millenium, 368-382.

[6] Galasso, F.S. (1969) Structure, Properties and Preparation of Perovskite Type Compounds. Pergamon Press, Oxford.

[7] Cullity, B.D. (1978) Elements of X-Ray Diffraction. 2nd Edition, Addison-Wesley Publishing Company Inc., Phillippines.

[8] West, A.R. (1974) Solid State Chemistry and It’s Applications. Wiley, New York.

[9] Xie, Q. and McCourt, F. (2008) Nanotechnology Engineering NE 320L Lab Manual. University of Waterloo, Waterloo, 35-39.

[10] So, W.W., Jang, J.S., Rhee, Y.W., Kim, K.J. and Moon, S.J. (2001) Preparation of Nanosized Crystalline CdS Particles by the Hydrothermal Treatment. Journal of Colloid and Interface Science, 237, 136-141. http://dx.doi.org/10.1006/jcis.2001.7489

[11] Ghosh, S.C., Thanachayanont, C. and Dutta, J. (2014) Studies on Zinc Sulphide Nanoparticles for Field Emission Devices. The 1st ECTI Annual Conference (ECTI-CON2004), Pattaya, 13-14 May 2004, 145-148.

[12] Williamson, G.K. and Hall, W.H. (1953) X-Ray Line Broadening from Filed Aluminium and Wolfram. Acta Metallurgica, 1, 22-31. http://dx.doi.org/10.1016/0001-6160(53)90006-6 
Scientific Research Publishing (SCIRP) is one of the largest Open Access journal publishers. It is currently publishing more than 200 open access, online, peer-reviewed journals covering a wide range of academic disciplines. SCIRP serves the worldwide academic communities and contributes to the progress and application of science with its publication.

Other selected journals from SCIRP are listed as below. Submit your manuscript to us via either submit@scirp.org or Online Submission Portal.
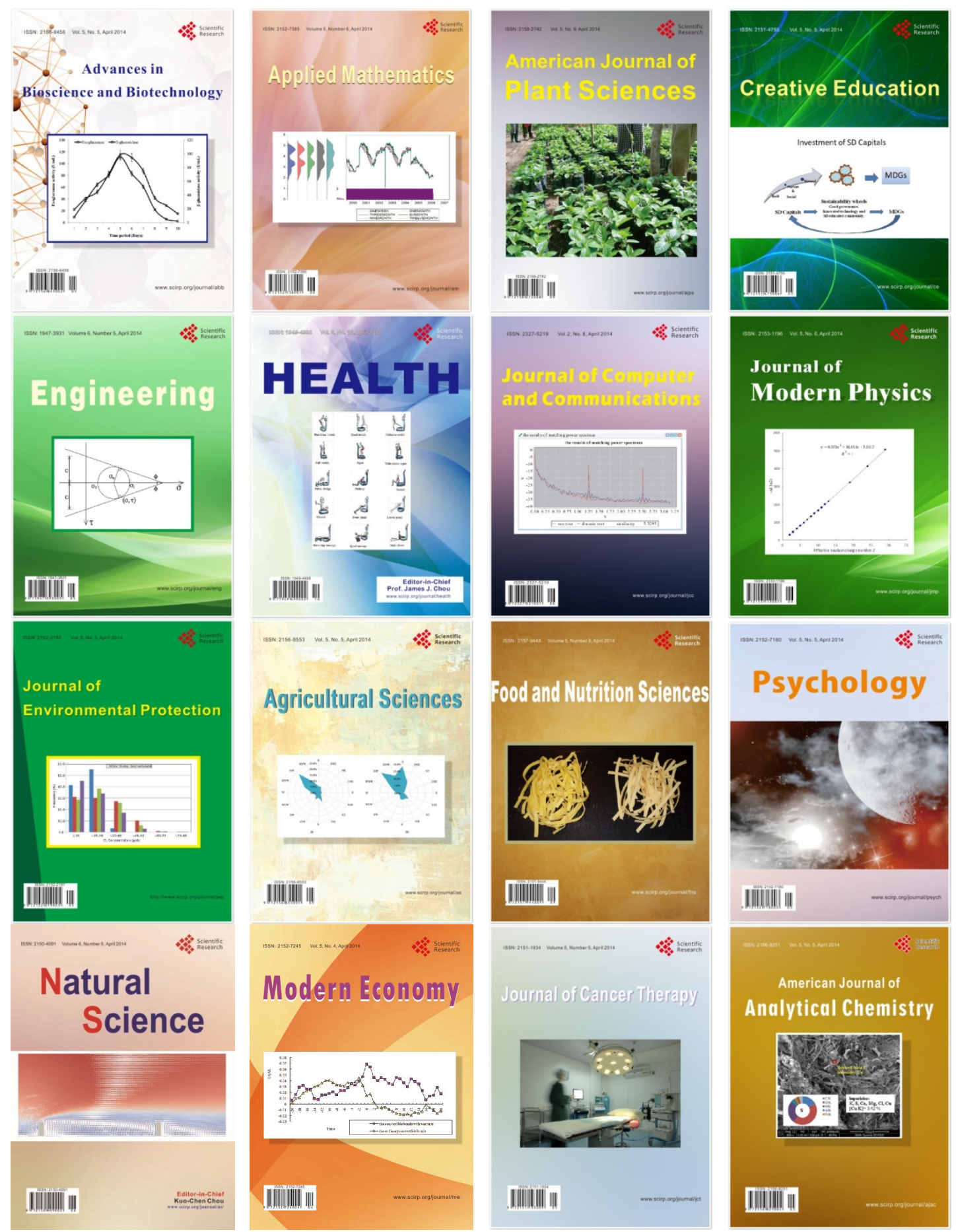